\title{
Infections with Digenetic Trematode Metacercariae in Freshwater Fishes from Two Visiting Sites of Migratory Birds in Gyeongsangnam-do, Republic of Korea
}

\author{
Woon-Mok Sohn*, Byoung-Kuk Na \\ Department of Parasitology and Tropical Medicine, and Institute of Health Sciences, Gyeongsang National University College of Medicine, \\ Jinju 52727, Korea
}

\begin{abstract}
The infection status of digenetic trematode metacercariae (DTM) was investigated in fishes from 2 representative visiting sites of migratory birds in Gyeongsangnam-do, the Republic of Korea (Korea). A totaly 220 freshwater fishes (7 species) were collected from Junam-jeosuji (reservoir), and 127 fishes (7 species) were also collected from Woopo-neup (swamp) in June and October 2017. As the control group, total 312 fish (22 spp.) from Yangcheon in Sancheong-gun, Gyeongsangnam-do were also collected in June and October 2017. All fishes collected in 3 sites were examined with the artificial digestion method. In the fishes from Junam-jeosuji, more than 4 species, i.e., Clonorchis sinensis, Echinostoma spp., Diplostomum spp. and Cyathocotyle orientalis, of DTM were detected and their endemicy was very low, 0.70 . More than 6 species, i.e., C. sinensis, Echinostoma spp., Metorchis orientalis, Clinostomum complanatum, Diplostomum spp. and C. orientalis, of DTM were found in the fishes from Woopo-neup, and their endemicy was low, 5.16. In the fishes from Yangcheon, more than 8 species, i.e., C. sinensis, Metagonimus spp., Centrocestus armatus, C. complanatum, C. orientalis, M. orientalis, Echinostoma spp., and Diplostomum spp., of DTM were detected, and their endemicity was relatively high, 95.48. The percentages of avian trematode metacercariae (ATM) were $99.6 \%$ and $94.7 \%$ in fishes from Junam-jeosuji and Woopo-neup whereas it was $74.1 \%$ in the control site, Yangcheon. The above findings suggested that migratory birds partly affect in endemicity of DTM in the fish in the 2 visiting sites in Gyeongsangnam-do, Korea.
\end{abstract}

Key words: Migratory bird, digenetic trematode, metacercariae, Junam-jeosuji, Woopo-neup, Yangcheon

\section{INTRODUCTION}

Numerous species of avian trematodes have been reported in the Republic of Korea (Korea). Most of them are the members of family Echinostomatidae, Microphallidae, and Gymnophallidae. More than 14 species of echinostomatid flukes, i.e., Acanthoparyphium marilae, A. tyosenense, Echinochasmus japonicus, Echinoparyphium recurvatum, Echinostoma gotoi, E. miyagawai, E. revolutum, Himasthla kusasigi, Patagifer bilobus, Petasiger neocomense, Saakotrema metatestis, Pegosomum bubulcum, Nephrostomum ramosum and Chaunocephalus ferox, were detected from the various species of bird in Korea [1-10]. Total 6 species of microphallid (Microphallus koreana, Gynaecotyla squatarolae, Maritrema jebuensis, M. obstipum, Endocotyle incana

\footnotetext{
- Received 28 March 2019, revised 25 May 2019, accepted 27 May 2019.

*Corresponding author (wmsohn@gnu.ac.kr)

(C) 2019, Korean Society for Parasitology and Tropical Medicine

This is an Open Access article distributed under the terms of the Creative Commons

Attribution Non-Commercial License (http://creativecommons.org/licenses/by-nc/4.0) which permits unrestricted non-commercial use, distribution, and reproduction in any

medium, provided the original work is properly cited.
}

and Spelotrema pseudogonocotyla) [11-14], and 4 species of gymnophallid flukes (Gymnophalloides seoi, Gymnophallus macrostoma, Parvatrema duboisi and P. homoeotecnum) [15-17] were reported as avian trematodes in Korea. On the other hand, it has been known that several species of avian trematode, i.e., Centrocestus armatus, Cyathocotyle orientalis, Holostephanus nipponicus, Clinostomum complanatum, Metorchis orientalis, M. taiwanensis and Diplostomum spp., metacercariae are frequently detected in the freshwater fishes in Korea [18-21].

Junam-jeosuji (a reservoir in Dong-eup, Changwon-si) and Woopo-neup (a swamp in Changnyung-gun) in Gyeongsangnam-do, are famous as the visiting sites of miratory birds in Winter season. More than 20 species of migratory birds from China, Siberia and so on winter until late in March [22]. The specific ecological condition, such as the massive visiting of various migratory birds, may be affected the avian trematode fauna in 2 sites. On the other hand, many Korean workers have been investigated the infection status with digenetic trematode metacercariae (DTM) including Clonorchis sinensis in fishes from various ecological environments, such as river, 
stream, lake, pond, swamp and even coast, to estimate the DTM endemicities [18-21,23-29]. Most of these studies were performed on the specific trematode species related with human infections, such as C. sinensis, heterophyid including Metagonimus yokogawai and echinostomatid flukes, in Korea [23-29]. However, there have been not so many studies on the avian trematode metacercariae (ATM) in fishes from the visiting sites of migratory birds in Korea. Moreover, the infection status of DTM in fishes from Junam-jeosuji and Woopo-neup was surveyed in a long time ago [30-32]. Therefore, we are going to focus on the infection status of ATM in 2 representative visiting sites of migratory birds, Junam-jeosuji and Woopo-neup, with a comparison of that in control site, Yangcheon, in Gyeongsangnam-do, Korea.

\section{MATERIALS AND METHODS}

Main survey (fish collection) sites were Junam-jeosuji in Dong-eup (eup = town) $(35.313588 ; 128.666354)$, Changwonsi (si=city), Gyeongsangnam-do (do=province), and Wooponeup in Ibang-myeon (myeon = township) (35.566238; 128.408215), Changnyeong-gun (gun= county), Gyeongsangnam-do, Korea. Total 220 fishes (7 spp.) from Junam-jeosuji were examined in June and October 2017. Fish species (No. of fish) examined were Erythroculter erythropterus (52), Lepomis macrochirus (52), Hemiculter eigenmanni (46), Cyprinus carpio (26), Acanthorhodeus gracilis (20), Carassius auratus (12), Micropterus salmoides (12). A total of 127 fishes (7 spp.) from Woopo-neup were examined in October 2017. Fish species (No. of fish) examined were Pseudorasbora parva (40), L. macrochirus (28), C. auratus (20), E. erythropterus (16), M. salmoides (15), H. eigenmanni (6), C. carpio (2).

As the control group, total 312 fish (22 spp.) from Yangcheon in Saengbiryang-myeon (35.355960; 128.056635), Sancheong-gun, Gyeongsangnam-do were also examined in June and October 2017. Fish species (No. of fish) examined were $M$. salmoides (36), Acheilognathus yamatsutae (31), Acheilognathus majusculus (30), Zacco platypus (27), L. macrochirus (25), C. auratus (21), Zacco temminckii (20), A. gracilis (20), Zacco koreanus (20), Pseudogobio esocinus (15), Squalidus japonicus coreanus (15), Pungtungia herzi (12), Coreoperca herzi (9), Acheilognathus rhombeus (7), Hemibarbus labeo (6), Hemibarbus longirostris (5), Odontobutis platycephala (4), Squalidus gracilis majimae (3), Acheilognathus koreensis (2), Acanthorhodeus macropterus (2), Sarcocheilichthys nigripinnis morii (1), Sarcocheilichthys variegatus wakiyae (1).

All collected fishes with ice were transferred to the laboratory of the Department of Parasitology and Tropical Medicine, Gyeongsang National University College of Medicine, Jinju, Korea. After the identification of fish species, they were individually ground with a mortar. Each ground fish meat was mixed with artificial gastric juice and the mixture was incubated at $36^{\circ} \mathrm{C}$ for about $2 \mathrm{hr}$. The digested material was filtered with $1 \times 1 \mathrm{~mm}$ of mesh and washed with $0.85 \%$ saline untill the supernatant is clear. The sediment was carefully examined under a stereomicroscope. Digenetic trematode metacercariae (DTM) except for 3 species, i.e., Metacercaria hasegawai, Exorchis oviformis and Pseudoexorchis major, were separately collected by the general features [33], and they were counted to get hold of infection rates (\%) and intensities (No. of DTM per fish infected) by fish species.

The endemicity of DTM was calculated by the Positive Rate in Fish Species (PRFS: No. of fish species infected/Total No. of fish species examined) $\times$ the Positive Rate in Positive Fish Species (PRPFS: No. of positive fish/total No. of fish in positive fish species examined) $\times$ the Intensity of Metacercariae Per a Positive Fish (IMPPF: Total No. of metacercariae detected/total No. of positive fish). The endemicity of avian trematode metacercariae (ATM) was calculated by the endemicity of ATM/total endemicity of DTM by the surveyed site $\times 100$. More than 7 species, i.e., C. armatus, C. complanatum, C. orientalis, M. orientalis, M. taiwanensis Echinostoma spp. and Diplostomum spp., of DTM were treated as the ATM in this study.

\section{RESULTS}

In fishes from Junam-jeosuji, more than 4 species, i.e., C. sinensis, Echinostoma spp., Diplostomum sp. and C. orientalis, of DTM were detected, and their infection status by the fish species is detailedly shown in Table 1. More than 6 species, i.e., $C$. sinensis, Echinostoma spp., Diplostomum spp., C. orientalis, M. orientalis and C. complanatum, of DTM were found in fishes from Woopo-neup, and their infection status by the fish species is detailedly designated in Table 2. Unidentified echinostomatid metacercariae were dominant and commonly detected in all 7 fish species from 2 survey sites. Most of them were elliptical, 138-210 (162) × 103-150 (127) $\mu \mathrm{m}$ in size and had a head crown equipped with 27 collar spines including 4 end-group ones in each side like those of Isthmiophora hortensis (Fig. 1).

In fishes from Yangcheon (control site), more than 8 spe- 
Table 1. Infection status of trematode metacercariae by fish species caught from Junam-jeosuji (reservoir) in Changwon-si, Gyeongsangnam-do, Korea

\begin{tabular}{|c|c|c|c|c|}
\hline \multirow{2}{*}{ Trematode Fish sp. infected } & \multirow{2}{*}{ No. of fish examined } & \multirow{2}{*}{ No. of fish infected (\%) } & \multicolumn{2}{|c|}{ No. of metacercariae detected } \\
\hline & & & Range & Average \\
\hline \multicolumn{5}{|l|}{ Clonorchis sinensis } \\
\hline Hemiculter eigenmanni & 46 & $1(2.2)$ & - & 1.0 \\
\hline \multicolumn{5}{|l|}{ Echinostoma spp. } \\
\hline Erythroculter erythropterus & 52 & 7 (13.5) & $1-103$ & 16.6 \\
\hline Lepomis macrochirus & 52 & $19(36.5)$ & $1-10$ & 2.6 \\
\hline Hemiculter eigenmanni & 46 & $8(17.4)$ & $1-7$ & 2.6 \\
\hline Cyprinus carpio & 26 & $4(15.4)$ & $1-6$ & 2.3 \\
\hline Acanthorhodeus gracilis & 20 & $19(95.0)$ & $1-49$ & 10.7 \\
\hline Carassius auratus & 12 & $9(75.0)$ & $1-14$ & 8.3 \\
\hline Micropterus salmoides & 12 & $11(91.7)$ & $1-103$ & 8.7 \\
\hline Subtotal & 220 & $77(35.0)$ & $1-103$ & 8.7 \\
\hline \multicolumn{5}{|l|}{ Diplostomum spp. } \\
\hline Erythroculter erythropterus & 52 & $5(9.6)$ & $1-3$ & 1.6 \\
\hline Lepomis macrochirus & 52 & 1 (1.9) & - & 1.0 \\
\hline Hemiculter eigenmanni & 46 & $10(21.7)$ & $1-16$ & 3.6 \\
\hline Acanthorhodeus gracilis & 20 & $1(5.0)$ & - & 1.0 \\
\hline Subtotal & 170 & $17(10.0)$ & $1-16$ & 2.7 \\
\hline \multicolumn{5}{|l|}{ Cyathocotyle orientalis } \\
\hline Hemiculter eigenmanni & 46 & $2(4.3)$ & $1-2$ & 1.5 \\
\hline
\end{tabular}

Table 2. Infection status of trematode metacercariae by fish species caught Woopo-neup (swamp) in Changnyung-gun, Gyeongsangnam-do, Korea

\begin{tabular}{|c|c|c|c|c|}
\hline \multirow{2}{*}{ Trematode fish sp. infected } & \multirow{2}{*}{ No. of fish examined } & \multirow{2}{*}{ No. of fish infected (\%) } & \multicolumn{2}{|c|}{ No. of metacercariae detected } \\
\hline & & & Range & Average \\
\hline $\begin{array}{l}\text { Clonorchis sinensis } \\
\text { Pseudorasbora parva } \\
\text { Hemiculter eigenmanni } \\
\text { Subtotal }\end{array}$ & $\begin{array}{r}40 \\
6 \\
46\end{array}$ & $\begin{array}{r}11(27.5) \\
2(33.3) \\
13(28.3)\end{array}$ & $\begin{array}{c}1-17 \\
- \\
1-17\end{array}$ & $\begin{array}{l}4.6 \\
1.0 \\
4.1\end{array}$ \\
\hline $\begin{array}{l}\text { Echinostoma spp. } \\
\text { Pseudorasbora parva } \\
\text { Lepomis macrochirus } \\
\text { Carassius auratus } \\
\text { Erythroculter erythropterus } \\
\text { Micropterus salmoides } \\
\text { Hemiculter eigenmanni } \\
\text { Cyprinus carpio } \\
\text { Subtotal }\end{array}$ & $\begin{array}{r}40 \\
28 \\
20 \\
16 \\
15 \\
6 \\
2 \\
127\end{array}$ & $\begin{array}{c}38(95.0) \\
20(71.4) \\
18(90.0) \\
13(81.3) \\
13(86.7) \\
4(66.7) \\
2(100) \\
108(85.0)\end{array}$ & $\begin{array}{c}1-145 \\
1-9 \\
1-58 \\
1-25 \\
1-23 \\
1-6 \\
125-132 \\
1-145\end{array}$ & $\begin{array}{r}25.7 \\
3.1 \\
17.4 \\
10.2 \\
10.1 \\
3.0 \\
128.5 \\
17.5\end{array}$ \\
\hline $\begin{array}{l}\text { Metorchis orientalis } \\
\text { Pseudorasbora parva } \\
\text { Micropterus salmoides } \\
\text { Subtotal }\end{array}$ & $\begin{array}{l}40 \\
15 \\
55\end{array}$ & $\begin{array}{c}30(75.0) \\
1(6.7) \\
31(56.4)\end{array}$ & $\begin{array}{l}1-11 \\
- \\
1-11\end{array}$ & $\begin{array}{l}2.7 \\
2.0 \\
2.7\end{array}$ \\
\hline $\begin{array}{l}\text { Clinostomum complanatum } \\
\text { Pseudorasbora parva }\end{array}$ & 40 & $3(7.5)$ & $1-2$ & 1.7 \\
\hline $\begin{array}{l}\text { Diplostomum spp. } \\
\text { Pseudorasbora parva } \\
\text { Lepomis macrochirus } \\
\text { Carassius auratus } \\
\text { Erythroculter erythropterus } \\
\text { Hemiculter eigenmanni } \\
\text { Cyprinus carpio } \\
\text { Subtotal }\end{array}$ & $\begin{array}{r}40 \\
28 \\
20 \\
16 \\
6 \\
2 \\
112\end{array}$ & $\begin{aligned} & 28(70.0) \\
& 1(3.6) \\
& 3(15.0) \\
& 8(50.0) \\
& 2(33.3) \\
& 1(50.0) \\
& 43(38.4)\end{aligned}$ & $\begin{array}{c}1-7 \\
- \\
1-4 \\
1-5 \\
- \\
- \\
1-7\end{array}$ & $\begin{array}{l}2.4 \\
2.0 \\
2.3 \\
2.4 \\
1.0 \\
6.0 \\
2.4\end{array}$ \\
\hline $\begin{array}{l}\text { Cyathocotyle orientalis } \\
\text { Pseudorasbora parva } \\
\text { Micropterus salmoides } \\
\text { Hemiculter eigenmanni } \\
\text { Subtotal }\end{array}$ & $\begin{array}{r}40 \\
15 \\
6 \\
61\end{array}$ & $\begin{aligned} 40 & (100) \\
3 & (20.0) \\
1 & (16.7) \\
44 & (72.1)\end{aligned}$ & $\begin{array}{l}7-252 \\
1-6 \\
- \\
1-252\end{array}$ & $\begin{array}{r}60.1 \\
2.7 \\
1.0 \\
54.8\end{array}$ \\
\hline
\end{tabular}



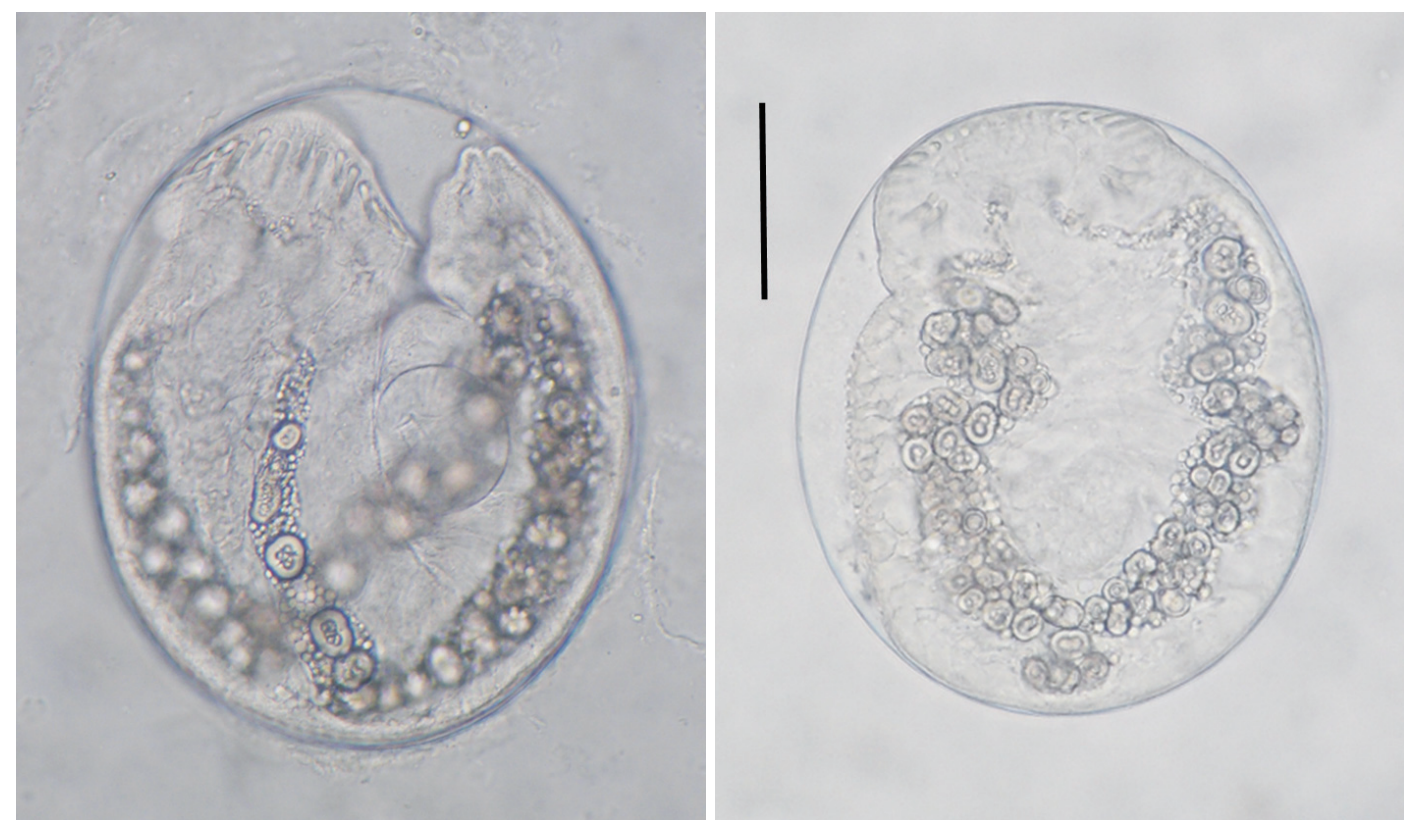

Fig. 1. Two echinostomatid metacercariae detected in a false dace, Pseudorasbora parva, from Woopo-neup in Changnyeong-gun, Gyeongsangnam-do, Korea. They were elliptical, 138-210 (162)×103-150 (127) $\mu \mathrm{m}$ in size and had a head crown equipped with 27 collar spines including 4 end-group ones in each sides. Scale bar is $50 \mu \mathrm{m}$.

cies, i.e., C. sinensis, Metagonimus spp., C. armatus, C. complanatum, C. orientalis, M. orientalis, Echinostoma spp., and Diplostomum spp., of DTM were detected, and their infection status by the fish species is detailedly revealed in Table 3.

The overall DTM endemicies were 0.70 and 5.16 in fishes from Junam-jeosuji and Woopo-neup, whereas it was 95.48 in the control site, Yangcheon. In Junam-jeosuji, the endemicity of Echinostoma spp. (3.045) was most high and those of remains were relatively very low. In Woopo-neup, the endemicities of C. orientalis (16.950) and Echinostoma spp. (14.875) was relatively high and those of remains were below 1.0. Whereas in Yangcheon, the endemicity of C. armatus (531.311) was very high, those of C. sinensis (23.408) and C. orientalis (21.028) were moderate levels and those of remains were relatively low. The endemicities with DTM by the surveyed localities are detailedly designated in Table 4 . The percentages of ATM were $99.6 \%$ and $94.7 \%$ in fishes from Junam-jeosuji and Wooponeup, whereas it was $74.1 \%$ in the control site, Yangcheon.

\section{DISCUSSION}

The number of fish species examined were not so enough to evaluate DTM infections in 2 survey sites in this study. Among 7 fish species (total No. of fish) examined in each surveyed sites, 6 ones, i.e., L. macrochirus (80), E. erythropterus (68), $H$. eigenmanni (52), C. auratus (32), C. carpio (28), and M. salmoides (27), were commonly investigated, and remain each one was A. gracilis (20) in Junam-jeosuji and $P$. parva (40) in Woopo-neup. Therefore, in this study, we examined total 347 fish in 8 species from 2 visiting sites of migratory birds and a total of 312 fish in 22 species from the control site, Yangcheon, in Sancheong-gun, Gyeongsangnam-do. The smaller number of fish species in the 2 main survey sites than in the control site is strongly suggested that the ecology for fish is not so good in 2 survey sites, Junam-jeosuji and Woopo-neup. The bad condition of fish ecology was revealed in the previous studies. Han et al. [30] examined total 7 fish species, P. parva (301), E. erythropterus (113), Rhodeus uyekii (44), Rhodeus ocellatus (9), Gnathopogon atromaculatus (= G. striatus) (6), C. carassius (=C. auratus) (2) and Acanthorhodeus taenianalis (=A. macropterus) (2), from Junam-jeosuji in 1992. Kong and Choi [31] investigated DTM infections in 8 fish species, G. atromaculatus (61), A. taenianalis (33), P. parva (29), C. carassius (22), Zacco platypus (20), Microphysogobio koreesis (19), R. uyekii (16) and Zacco temminckii (8), from Woopo-neup. Sohn and Choi [32] also examined on the infection status of DTM in 5 fish species, H. eigenmanni (45), P. parva (25), E. erythropterus (20), A. macropterus (20), and C. auratus (20), from Junam-jeosuji. The exotic 
Table 3. Infection status of trematode metacercariae by fish species caught from Yangcheon (stream) in Sancheong-gun, Gyeongsangnam-do, Korea

\begin{tabular}{|c|c|c|c|c|}
\hline \multirow{2}{*}{ Trematode fish sp. infected } & \multirow{2}{*}{ No. of fish examined } & \multirow{2}{*}{ No. of fish infected (\%) } & \multicolumn{2}{|c|}{ No. of metacercariae detected } \\
\hline & & & Range & Average \\
\hline \multicolumn{5}{|l|}{ Clonorchis sinensis } \\
\hline Acheilognathus yamatsutae & 31 & $21(67.7)$ & $1-12$ & 3.3 \\
\hline Acheilognathus majusculus & 30 & $16(53.3)$ & $1-10$ & 2.6 \\
\hline Zacco platypus & 27 & $4(14.8)$ & $1-2$ & 1.8 \\
\hline Lepomis macrochirus & 25 & $1(4.0)$ & - & 1.0 \\
\hline Acanthorhodeus gracilis & 20 & $18(90.0)$ & $1-59$ & 17.1 \\
\hline Pseudogobio esocinus & 15 & $15(100)$ & $11-124$ & 37.9 \\
\hline Squalidus japonicus coreanus & 15 & $15(100)$ & $15-364$ & 133.7 \\
\hline Pungtungia herzi & 12 & $12(100)$ & $40-45$ & $40-451$ \\
\hline Acheilognathus rhombeus & 7 & $6(85.7)$ & $1-34$ & 11.8 \\
\hline Hemibarbus labeo & 6 & $5(83.3)$ & $1-6$ & 2.4 \\
\hline Hemibarbus longirostris & 5 & $4(80.0)$ & $1-8$ & 2.8 \\
\hline Squalidus gracilis majimae & 3 & $3(100)$ & $32-195$ & 86.7 \\
\hline Acheilognathus koreensis & 2 & $2(100)$ & $1-3$ & 2.0 \\
\hline Acanthorhodeus macropterus & 2 & $2(100)$ & $4-7$ & 5.5 \\
\hline Sarcocheilichthys nigripinnis & 1 & $1(100)$ & - & 172.0 \\
\hline Sarcocheilichthys variegatus & 1 & $1(100)$ & - & 208.0 \\
\hline Subtotal & 202 & $126(62.4)$ & $1-451$ & 51.6 \\
\hline \multicolumn{5}{|l|}{ Metagonimus spo } \\
\hline Micropterus salmoides & 36 & $2(5.6)$ & - & 1.0 \\
\hline Acheilognathus yamatsutae & 31 & $4(12.9)$ & - & 1.0 \\
\hline Zacco platypus & 27 & $15(55.6)$ & $1-17$ & 3.2 \\
\hline Lepomis macrochirus & 25 & $13(52.0)$ & $1-11$ & 2.5 \\
\hline Carassius auratus & 21 & $21(100)$ & $2-39$ & 14.4 \\
\hline Zacco temminckii & 20 & $11(55.0)$ & $1-3$ & 1.6 \\
\hline Acanthorhodeus gracilis & 20 & $5(25.0)$ & $1-2$ & 1.4 \\
\hline Zacco koreanus & 20 & $15(75.0)$ & $1-10$ & 3.8 \\
\hline Squalidus japonicus coreanus & 15 & $6(40.0)$ & $1-4$ & 1.8 \\
\hline Pungtungia herzi & 12 & $7(58.3)$ & $1-6$ & 1.9 \\
\hline Hemibarbus longirostris & 5 & $2(40.0)$ & $1-3$ & 2.0 \\
\hline Squalidus gracilis majimae & 3 & $3(100)$ & $1-7$ & 3.3 \\
\hline Sarcocheilichthys nigripinnis & 1 & $1(100)$ & $-1-1$ & $\begin{array}{l}0.0 \\
1.0\end{array}$ \\
\hline Sarcocheilichthys variegatus & 1 & $1(100)$ & - & 1.0 \\
\hline Subtotal & 237 & $106(44.7)$ & $1-39$ & 4.8 \\
\hline \multicolumn{5}{|l|}{ Centrocestus armatus } \\
\hline Micropterus salmoides & 36 & $1(2.8)$ & - & 1.0 \\
\hline Acheilognathus yamatsutae & 31 & $6(19.4)$ & $1-2$ & 1.2 \\
\hline Zacco platypus & 27 & $27(100)$ & $526-9,860$ & 3,330 \\
\hline Carassius auratus & 21 & 7 (33.3) & $1-6$ & 2.7 \\
\hline Zacco temminckii & 20 & $20(100)$ & $252-7,315$ & 2,738 \\
\hline Acanthorhodeus gracilis & 20 & $6(30.0)$ & $1-17$ & 5.3 \\
\hline Zacco koreanus & 20 & $20(100)$ & $1,030-17,330$ & 5,555 \\
\hline Squalidus japonicus coreanus & 15 & $1(6.7)$ & - & 1.0 \\
\hline Acheilognathus rhombeus & $1(14.3)$ & $1(14.3)$ & - & 1.0 \\
\hline Subtotal & 197 & $89(45.2)$ & $1-17,330$ & 2,874 \\
\hline \multicolumn{5}{|l|}{ Echinostoma spp. } \\
\hline Micropterus salmoides & 36 & $10(27.8)$ & $1-16$ & 4.0 \\
\hline Acheilognathus yamatsutae & 31 & $2(6.5)$ & - & 1.0 \\
\hline Acheilognathus majusculus & 30 & $1(3.3)$ & - & 2.0 \\
\hline Acanthorhodeus gracilis & 20 & $8(40.0)$ & $1-6$ & 1.9 \\
\hline Pseudogobio esocinus & 15 & $1(6.7)$ & - & 5.0 \\
\hline Squalidus japonicus coreanus & 15 & $2(13.3) 0$ & $2-4$ & 3.0 \\
\hline Pungtungia herzi & 12 & $3(25.0)$ & $2-12$ & 6.7 \\
\hline Acheilognathus rhombeus & 7 & $2(28.6)$ & $-2-12$ & $\begin{array}{l}0.1 \\
1.0\end{array}$ \\
\hline Hemibarbus longirostris & 5 & $3(60.0)$ & $1-2$ & 1.3 \\
\hline Subtotal & 171 & $32(18.7)$ & $1-16$ & 3.0 \\
\hline
\end{tabular}


Table 3. Continued

\begin{tabular}{|c|c|c|c|c|}
\hline \multirow{2}{*}{ Trematode fish sp. infected } & \multirow{2}{*}{ No. of fish examined } & \multirow{2}{*}{ No. of fish infected (\%) } & \multicolumn{2}{|c|}{ No. of metacercariae detected } \\
\hline & & & Range & Average \\
\hline \multicolumn{5}{|l|}{ Clinostomum complanatum } \\
\hline Acheilognathus yamatsutae & 31 & $23(74.2)$ & $1-28$ & 5.6 \\
\hline Acheilognathus majusculus & 30 & $23(76.7)$ & $1-52$ & 17.1 \\
\hline Zacco platypus & 27 & $6(22.2)$ & $1-15$ & 7.7 \\
\hline Carassius auratus & 21 & $1(4.8)$ & - & 1.0 \\
\hline Zacco temminckii & 20 & $5(25.0)$ & $1-7$ & 2.6 \\
\hline Acanthorhodeus gracilis & 20 & $10(50.0)$ & $16-253$ & 57.1 \\
\hline Zacco koreanus & 20 & $9(45.0)$ & $1-14$ & 7.7 \\
\hline Squalidus japonicus coreanus & 15 & $15(100)$ & $1-108$ & 31.4 \\
\hline Pungtungia herzi & 12 & $11(91.7)$ & $1-73$ & 19.2 \\
\hline Acheilognathus rhombeus & 7 & $3(42.9)$ & $4-50$ & 24.0 \\
\hline Hemibarbus labeo & 6 & $2(33.3)$ & $2-5$ & 3.5 \\
\hline Hemibarbus longirostris & 5 & $3(60.0)$ & - & 1.0 \\
\hline Squalidus gracilis majimae & 3 & $1(33.3)$ & - & 1.0 \\
\hline Acheilognathus koreensis & 2 & $2(100)$ & $7-33$ & 20.0 \\
\hline Acanthorhodeus macropterus & 2 & $2(100)$ & $8-9$ & 8.5 \\
\hline Subtotal & 221 & $116(52.5)$ & $1-253$ & 17.6 \\
\hline \multicolumn{5}{|l|}{ Metorchis orientalis } \\
\hline Acheilognathus majusculus & 30 & $2(6.7)$ & $1-2$ & 1.5 \\
\hline Acanthorhodeus gracilis & 20 & $1(5.0)$ & - & 1.0 \\
\hline Pseudogobio esocinus & 15 & $2(13.3)$ & - & 1.0 \\
\hline Pungtungia herzi & 12 & $9(75.0)$ & $1-8$ & 3.4 \\
\hline Subtotal & 77 & $14(18.2)$ & $1-8$ & 2.6 \\
\hline \multicolumn{5}{|l|}{ Diplostomum spp. } \\
\hline Acheilognathus yamatsutae & 31 & $5(16.1)$ & $1-2$ & 1.2 \\
\hline Acheilognathus majusculus & 30 & $3(10.0)$ & $1-2$ & 1.3 \\
\hline Zacco temminckii & 20 & $1(5.0)$ & - & 1.0 \\
\hline Acanthorhodeus gracilis & 20 & $1(5.0)$ & - & 1.0 \\
\hline Pseudogobio esocinus & 15 & $5(33.3)$ & $1-5$ & 2.8 \\
\hline Squalidus japonicus coreanus & 15 & $4(26.7)$ & $1-6$ & 3.0 \\
\hline Acheilognathus rhombeus & 7 & $1(14.3)$ & - & 1.0 \\
\hline Acheilognathus koreensis & 2 & $2(100)$ & - & 1.0 \\
\hline Subtotal & 140 & $22(15.7)$ & $1-6$ & 1.9 \\
\hline \multicolumn{5}{|l|}{ Cyathocotyle orientalis } \\
\hline Acheilognathus yamatsutae & 31 & $22(71.0)$ & $1-46$ & 7.5 \\
\hline Acheilognathus majusculus & 30 & $23(76.7)$ & $1-35$ & 8.4 \\
\hline Zacco platypus & 27 & $6(22.2)$ & $1-4$ & 2.5 \\
\hline Lepomis macrochirus & 25 & $1(4.0)$ & - & 1.0 \\
\hline Zacco temminckii & 20 & $18(90.0)$ & $1-10$ & 3.3 \\
\hline Acanthorhodeus gracilis & 20 & $13(65.0)$ & $1-24$ & 7.2 \\
\hline Zacco koreanus & 20 & $20(100)$ & $1-93$ & 31.2 \\
\hline Pseudogobio esocinus & 15 & $11(73.3)$ & $1-17$ & 5.7 \\
\hline Squalidus japonicus coreanus & 15 & 14 (93.3) & $1-57$ & 13.6 \\
\hline Pungtungia herzi & 12 & $12(100)$ & $3-1,698$ & 440.3 \\
\hline Acheilognathus rhombeus & 7 & $5(71.4)$ & $2-30$ & 10.4 \\
\hline Hemibarbus labeo & 6 & $2(33.3)$ & $1-2$ & 1.5 \\
\hline Hemibarbus longirostris & 5 & $3(60.0)$ & $1-86$ & 29.3 \\
\hline Acheilognathus koreensis & 2 & $2(100)$ & $1-7$ & 4.0 \\
\hline Sarcocheilichthys nigripinnis & 1 & $1(100)$ & - & 1.0 \\
\hline Sarcocheilichthys variegatus & $1(100)$ & $1(100)$ & - & 5.0 \\
\hline Subtotal & 237 & $154(65.0)$ & $1-1,698$ & 44.5 \\
\hline
\end{tabular}

predatory fish, L. macrochirus and M. salmoides, and the bigsized fish, E. erythropterus and H. eigenmanni, were dominant in this study. Whereas, all fish examined were the native fish species and P. parva and E. erythropterus were dominant in the previous studies [30-32].
In the calculating formula of DTM endemicity, PRFS (the positive rate in fish species) was originated without the consideration of host-specificity between trematode larvae (cercariae) and fish species. The list of fish host for each DTM has not been known yet in Korea except some human trematodes in- 
Table 4. Endemicity of digenetic trematode metacercariae in fishes from 3 subjected sites in Gyeongsangnam-do, Korea

\begin{tabular}{|c|c|c|c|c|}
\hline Locality/Trematode metacercariae & $\mathrm{PRFS}^{\mathrm{a}}$ & $\mathrm{PRPFS}^{\mathrm{b}}$ & IMPPF $^{c}$ & Endemicity $^{d}$ \\
\hline \multicolumn{5}{|l|}{ Junam-jeosuji } \\
\hline Clonorchis sinensis & 0.143 & 0.022 & 1.0 & 0.003 \\
\hline Echinostoma spp. & 1.0 & 0.35 & 8.7 & 3.045 \\
\hline Diplostomum spp. & 0.57 & 0.10 & 2.7 & 0.154 \\
\hline Cyathocotyle orientalis & 0.143 & 0.043 & 1.5 & 0.009 \\
\hline Subtotal & 0.46 & 0.201 & 7.4 & 0.70 \\
\hline \multicolumn{5}{|l|}{ Woopo-neup } \\
\hline Clonorchis sinensis & 0.286 & 0.283 & 4.1 & 0.332 \\
\hline Echinostoma spp. & 1.0 & 0.85 & 17.5 & 14.875 \\
\hline Diplostomum spp. & 0.857 & 0.384 & 2.4 & 0.790 \\
\hline Cyathocotyle orientalis & 0.429 & 0.721 & 54.8 & 16.950 \\
\hline Metorchis orientalis & 0.286 & 0.564 & 2.7 & 0.436 \\
\hline Clinostomum complanatum & 0.143 & 0.075 & 1.7 & 0.018 \\
\hline Subtotal & 0.50 & 0.549 & 18.8 & 5.16 \\
\hline \multicolumn{5}{|l|}{ Yangcheon } \\
\hline Clonorchis sinensis & 0.727 & 0.624 & 51.6 & 23.408 \\
\hline Metagonimus spp. & 0.636 & 0.447 & 4.8 & 1.365 \\
\hline Centrocestus armatus & 0.409 & 0.452 & 2,874 & 531.311 \\
\hline Echinostoma spp. & 0.409 & 0.187 & 3.0 & 0.229 \\
\hline Clinostomum complanatum & 0.682 & 0.525 & 17.6 & 6.302 \\
\hline Metorchis orientalis & 0.182 & 0.182 & 2.6 & 0.086 \\
\hline Diplostomum spp. & 0.364 & 0.157 & 1.9 & 0.109 \\
\hline Cyathocotyle orientalis & 0.727 & 0.650 & 44.5 & 21.028 \\
\hline Subtotal & 0.52 & 0.445 & 412.6 & 95.48 \\
\hline
\end{tabular}

aPRFS (Positive Rate of Fish Species/100: No. of fish species positive/total No. of fish species examined).

bPRPFS (Positive Rate in Positive Fish Species/100: No. of positive fish/total No. in positive fish species examined.

'IMPPF (Intensity of Metacercariae Per a Positive Fish: Total No. of metacercariae detected/total No. of positive fish.

daxbxc.

cluding C. sinensis. In this study, we evenly applied PRFS as a factor of all DTM endemicity regardless of the host-specificity. The DTM endemicity, PRFS $\times$ PRPFS $\times$ IMPPF, is thought to be the more reasonable index to compare the endemicities of individual trematode species and specific trematode groups in a certain survey site. Undoubtedly, it is not to be an absolute index, shows a kind of trend for DTM infections, and its standard is changeable according to the trematode species.

Among the 24 avian trematodes reported in Korea, microphallid and gymnophallid flukes have no possibility to infect via the consumption of freshwater fish. Microphallid flukes, i.e., M. koreana, G. squatarolae, M. jebuensis, M. obstipum, E. incana and $S$. pseudogonocotyla, are to be mainly infected with the eatings of crustacean intermediate hosts like crabs [11-14]. Gymnophallids, i.e., G. seoi, G. macrostoma, P. duboisi and P. homoeotecnum, are mainly infected in clam-eating birds [1517]. However, echinostomatid ones are known to be infected with the consumption of fish and molluscs including snails [1-10]. In this study, unidentified echinostomatid metacercariae were dominantly detected in all fish species from 2 survey sites. They were elliptical, $162 \times 127 \mu \mathrm{m}$ in average size and morphologically very similar with those of I. hortensis. The metacercariae of $I$. hortensis previously reported were globular or elliptical, 143-165 (154) by 128-158 (144) $\mu \mathrm{m}$ in size, and have 27 collar spines including 4 end group ones in each side of the head crown [29]. However, most of echinostomatid metacercariae detected were inactive in the cyst wall, and then we could not obtain the adult worms from hamsters experimentally infected with them. Reason why the echinostomatid metacercariae are dominant in fishes from 2 survey sites should be clarified with the recovery and identification of adults in near future.

The metacercariae of C. sinensis (CsMc) were detected in only 2 fish species, P. parva and H. eigenmanni from the 2 main survey sites and their prevalence and intensity were very low in this study. Han et al. [30] found them in 2 fish species, G. atromaculatus and $P$. parva, from Junam-jeosuji, Kong and Choi [31] detected CsMc in 4 fish species, G. atromaculatus P. parva, M. koreensis and A. taenianalis, from Woopo-neup and Sohn and Choi [32] also found them in 4 fish species, H. eigenmanni, P. parva, E. erythropterus and A. macropterus, from Junamjeosuji. In previous studies, there are some differences in prevalences and intensities of CsMc, but their endemicity is not so high in fishes from 2 survey sites. 
Conclusively, it was confirmed by the present study that the proportions of ATM are higher in fishes from 2 visiting sites of migratory birds, Junam-jeosuji and Woopo-neup, in Gyeongsangnam-do, Korea. However, overall DTM endemicies were very low in fishes from 2 survey sites when we compared with that in fishes from the control site, Yangcheon. These findings partly suggested that the specific ecological condition, the massive visiting of migratory birds, is to be affected the endemicity of avian trematodes. However, some environmental factors, i.e., the bad fish ecology with agitating of exotic fish species like blue gill and large mouth bass, a long-lasting drought and a remarkable propagation of water plants, may be acted as the limited points in this study.

\section{ACKNOWLEDGMENTS}

This study was supported by a grant from Korea Association of Health Promotion (KAHP-2017: Survey on the effects of migratory bird in the infection status of trematode metacercariae in fish from Junam Reservoir). We thank Jung-A Kim and Hee-Joo Kim (Department of Parasitology and Tropical Medicine, Gyeongsang National University College of Medicine, Jinju, Korea), for their help in the examination of fish.

\section{CONFLICT OF INTEREST}

The authors declare no conflict of interest related to this study.

\section{REFERENCES}

1. Issiki O. On the trematode, Echinostoma revolutum Fröohlich, 1802 from the Korean wild duck. Central Vet Med J (Japan) 1934; 47: 639-647 (in Japanese).

2. Yamaguti S. Studies on the helminth fauna of Japan. Part 25. Trematodes of birds, IV. Jpn J Zool 1939; 8: 131-210.

3. Chu JK, Cho YJ, Chung SB, Won BO, Yoon MB. Study on the trematode parasites of the birds in Korea. Korean J Parasitol 1973; 22: 70-75.

4. Eom KS, Rim HJ, Jang DH. A study on the parasitic helminths of domestic duck (Anas platyrhynchos var. domestica Linnaeus) in Korea. Korean J Parasitol 1984; 22: 215-221.

5. Ryang YS, Ahn YK, Yoon MB. Trematode infections in the small intestine of Egretta alba modesta in Kangwon-do. Korean J Parasitol 1991; 29: 227-223.

6. Chu JP, Cho YJ, Yo JC, Park SG. Himasthla kusasigi (Trematoda, Echinostomatidae) recovered from the intestine of the dulin,
Calidris alpina sakhalina, in Korea. Korean J Syst Zool 2000; 16: 125-131.

7. Chung OS, Joo KH, Lee WS. Acanthoparyphium tyosenense infection in great knots and turnstone on the western coast of Korea. J Ecol Field Biol 2007; 30: 97-99.

8. Choe SJ, Lee DM, Park HS, Oh MH, Jeon HK, Lee YS, Na KJ, Kim YJ, Lee H, Eom KS. Three Echinostome species from wild birds in the Republic of Korea. Korean J Parasitol 2014; 52: 513520.

9. Choe SJ, Lee DM, Park HS, Jeon HK, Lee YS, Kim EJ, Na KJ, Eom KS. Two Echinostome Species, Pegosomum bubulcum and Nephrostomum ramosum (Digenea: Echinostomatidae), from an eastern cattle egret, Bubulcus ibis coromandus, in Republic of Korea. Korean J Parasitol 2016; 54: 485-496.

10. Choe SJ, Lee DM, Park HS, Jeon HK, Lee YS, Na KJ, Park SR, Lee $\mathrm{H}$, Eom KS. A case of chaunocephalosis by Chaunocephalus ferox (Digenea: Echinostomatidae) in an oriental white stork, Ciconia boyciana, in Korea. Korean J Parasitol 2016; 54: 659-665.

11. Guk SM, Chai JY, Sohn WM, Kim YM, Sim S, Seo M. Microphallus koreana $\mathrm{n}$. sp. (Trematoda: Microphallidae) transmitted by a marine crab, Macrophthalmus dilatatus. Korean J Parasitol 2008; 46: 165-169.

12. Seo M, Guk SM, Chai JY. The ruddy turnstone, Arenaria interpres interpres, a new definitive host for Gynaecotyla squatarolae (Digenea: Microphallidae). Korean J Parasitol 2008; 46: 41-43.

13. Chung OS, Sohn WM, Chai JY, Seo M, Lee HJ. Discovery of Maritrema obstipum (Digenea: Microphallidae) from migratory birds in Korea. Korean J Parasitol 2011; 49: 457-460.

14. Yoo HJ, Chung OS, Seo M. Discovery of Endocotyle incana and Spelotrema pseudogonotyla (Digenea: Microphallidae) from scolopacid migratory birds in Korea. Korean J Parasitol 2012; 50: 273-276.

15. Yamaguti S. Studies on the helminth fauna of Japan. Part 25. Trematodes of birds, IV. Jap J Zool 1939; 8: 131-210.

16. Ryang YS, Yoo JC, Lee SH, Chai JY. The Palearctic oystercatcher Haematopus ostralegus, a natural definitive host for Gymnophalloides seoi. J Parasitol 2000; 86: 418-419.

17. Chung OS, Lee HJ, Sohn WM, Park YK, Chai JY, Seo M. Discovery of Parvatrema duboisi and Parvatrema homoeotecnum (Digenea: Gymnophallidae) from migratory birds in Korea. Korean J Parasitol 2010; 48: 271-274.

18. Chun SK. Studies on some trematodes whose intermediate hosts are fishes in the Naktong River. Bull Pusan Fish Coll 1962; 4: 21-38.

19. Rhee JK, Lee HI, Baek BK, Kim PG. Survey on encysted cercariae of trematodes from freshwater fishes in Mangyeong riverside area. Korean J Parasitol 1983; 21: 187-192.

20. Rhee JK, Rim MH, Baek BK, Lee HI. Survey on encysted cercariae of trematodes from freshwater fishes in Tongjin riverside areas in Korea. Korean J Parasitol 1984; 22: 190-202.

21. Sohn WM, Na BK, Cho SH, Lee SW, Choi SB, Seok WS. Trematode metacercariae in freshwater fish from water systems of Hantangang and Imjingang in Republic of Korea. Korean J Para- 
sitol 2015; 53: 289-298.

22. Junam-jeosuji and Woopo-neup in Gyeongnam Province (South Korea) in Wikipedia - The free ency`clopedia: http://en.wikipedia.org.

23. Sohn WM, Na BK, Cho SH, Park MY, Kim CH, Hwang MA, No KW, Yoon KB, Lim HC. Prevalence of Clonorchis sinensis metacercariae in fish from water systems of Seomjin-gang (River). Korean J Parasitol 2017; 55: 305-312.

24. Sohn WM, Na BK, Cho SH, Ju JW, Son DC. Prevalence and intensity of Clonorchis sinensis metacercariae in freshwater fish from Wicheon Stream in Gunwi-gun, Gyeongsangbuk-do, Korea. Korean J Parasitol 2018; 56: 43-50.

25. Yoon KB, Lim HC, Jeon DY, Park S, Cho SH, Ju JW, Shin SS, Na BK, Sohn WM. Infection status with Clonorchis sinensis metacercariae in fish from Tamjin-gang (River) in Jeollanam-do, Republic of Korea. Korean J Parasitol 2018; 56: 183-188.

26. Sohn WM, Na BK, Cho SH, Ju JW, Kim CH, Yoon KB. Infection status with Metagonimus spp. metacercariae in fishes from Seomjin-gang and Tamjin-gang in Republic of Korea. Korean J Parasitol 2018; 56: 351-358.

27. Sohn WM, Na BK, Cho SH, Ju JW, Lee SW, Seok WS. Infections with zoonotic trematode metacercariae in yellowfin goby, Acan- thogobius flavimanus, from coastal areas of Republic of Korea. Korean J Parasitol 2018; 56: 259-265.

28. Sohn WM, Na BK, Cho SH, Lee WJ. Prevalence and density of digenetic trematode metacercariae in clams and oysters from western coastal regions of the Republic of Korea. Korean J Parasitol 2017; 55: 399-408.

29. Sohn WM, Na BK, Cho SH, Ju JW. Infection status of Isthmiophora hortensis metacercariae in dark sleepers, Odontobutis species, from some water systems of the Republic of Korea. Korean J Parasitol 2018; 56: 633-637.

30. Han JY, Kong HH, Moon CH, Choi DW. Digenetic larval trematodes from freshwater fish in pond Junam. Kyungpook Univ Med J 1994; 35: 221-228.

31. Kong HH, Choi DW. Infection status of digenetic trematode larvae in freshwater fish in lake Upo, Kyungsangnam-do, Korea. Kyungpook Univ Med J 1994; 35: 167-173.

32. Sohn WM, Choi YS. Infection status with trematode metacercariae in the freshwater fish from Chunamchosuchi (pond), Uichang-gun, Kyongsangnam-do, Korea. Korean J Parasitol 1997; 35: 165-170 (in Korean).

33. Sohn WM. Fish-borne zoonotic trematode metacercariae in the Republic of Korea. Korean J Parasitol 2009; 47 (suppl): 103-113. 
